\title{
Differential somatic cell count-A novel method for routine mastitis screening in the frame of Dairy Herd Improvement testing programs
}

\author{
Malin Damm, ${ }^{1}$ Claus Holm, Mette Blaabjerg, Morten Novak Bro, and Daniel Schwarz ${ }^{1,2}$ \\ Foss Analytical A/S, Foss Allé 1, 3400 Hillerød, Denmark
}

\begin{abstract}
Somatic cell count (SCC) is used as key indicator in mastitis screening programs typically applied in the frame of Dairy Herd Improvement (DHI) testing programs. However, mastitis is still causing tremendous economic losses to the dairy industry. Hence, new biomarkers are needed that can be used for enhanced management of mastitis on dairy farms. Besides the determination of SCC, differentiation of cells has been described to be beneficial for a more definite description of the actual udder health status of dairy cows. The aim of this study was to develop a new method for rapid and simultaneous determination of SCC and a new parameter, differential somatic cell count (DSCC), in individual cow milk samples using flow cytometry. The method is sought to be applied in central milk testing laboratories, so that existing DHI infrastructures can be used. The DSCC represents the combined proportion of polymorphonuclear leukocytes (PMN) and lymphocytes expressed in percentage. The proportion of macrophages can be calculated by subtracting DSCC from 100\%. Our research revealed increasing proportions of PMN, but decreasing proportions of macrophages as SCC increased. However, lymphocytes occurred fairly constantly with low proportions across the entire SCC range. Hence, the DSCC parameter reflects the antidromic trend of PMN and macrophages. Fluorescence microscopy was used to evaluate the specificity of the new Foss DSCC method in terms of DSCC and a high correlation was found. Apart from that, the accuracy of cell differentiation using the Foss DSCC method was confirmed in a cell sorting trial. Total SCC could be determined equally well using the new method as compared with existing methods. The new method was further proven to be robust toward a range of method and milk-sample-related factors. In an
\end{abstract}

Received December 6, 2016.

Accepted February 2, 2017.

${ }^{1}$ These authors contributed equally.

${ }^{2}$ Corresponding author: das@foss.dk initial field trial, regular DHI samples of a local dairy herd were analyzed. The DSCC values occurred in a broad range from 34 to $79 \%$ in samples with $<400,000$ cells $/ \mathrm{mL}$. Higher DSCC values $(53-89 \%)$ were found in samples with $>400,000$ cells $/ \mathrm{mL}$. In conclusion, the new Foss DSCC method allows reliable, repeatable, fast, robust, and accurate determination of both DSCC and SCC at low cost. This, in turn, provides more accurate information on the actual udder health status of dairy cows. The practical application of DSCC in the frame of DHI testing programs, however, needs further investigation.

Key words: mastitis, udder health, differential somatic cell count, somatic cell count

\section{INTRODUCTION}

Mastitis is one of the most prevalent and costly diseases in the dairy cattle industry worldwide, and adversely affects dairy cow welfare as well (Menzies et al., 1995). A major problem of the spread and persistence of mastitis within dairy herds is subclinical mastitis, a condition where the udder and the milk appear normal although the mammary gland is inflamed, infected, or both. Subclinically infected cows act as a reservoir for bacteria, resulting in an unnoticeable spread of mastitis to healthy herd mates (Halasa et al., 2007). The effective control of subclinical mastitis can clearly result in large economic profit for dairy farmers (van den Borne et al., 2010). However, the effectiveness of control is highly dependent on how fast these cows are detected, and hence the efficacy of the udder health monitoring program (van den Borne et al., 2010).

Somatic cell counts in milk provide an indication of the inflammatory response in the mammary gland and hence a proxy for measuring IMI and milk quality at quarter, cow, herd, and population levels (Schukken et al., 2003). The optimal SCC cutoff point to distinguish between infected and uninfected at the individual cow level has been established at 200,000 cells/mL (IDF, 2013). Regular SCC testing and associated udder health monitoring programs have a substantial positive influence on single animals as well as the entire herd 
(Barkema et al., 1998). Nevertheless, a major need for new biomarkers that are specific for mastitis and easy to detect at an early stage of the disease has been described (Viguier et al., 2009).

Somatic cell count is a robust quantitative measurement, but does not divide the cells present in milk, which are mainly lymphocytes, macrophages, and PMN, into different cell types (Kehrli and Shuster, 1994; Rivas et al., 2001). These immune cells play an important role in inflammatory responses within the mammary gland (Paape et al., 1979; Sordillo and Nickerson, 1988). Lymphocytes regulate the induction and suppression of immune responses (Nickerson, 1989; Sordillo et al., 1997). Macrophages are active phagocytic cells capable of ingesting bacteria, cellular debris, and accumulated milk components (Sordillo et al., 1997). Moreover, they recognize invading pathogens and initiate an immune response (i.e., rapid recruitment of PMN into the mammary gland; Paape et al., 2002; Oviedo-Boyso et al., 2007). The main task of PMN is to defend against invading bacteria at the beginning of an acute inflammatory process (Paape et al., 1979; Oviedo-Boyso et al., 2007).

Due to the specific functions of the individual cell populations, the distribution of leukocytes differs between normal milk and mastitic milk (Nickerson, 1989). Specifically, proportions of PMN can reach up to $95 \%$ in milk from cows with mastitis (Paape et al., 1979; Kehrli and Shuster, 1994). In the uninfected mammary gland, however, SCC is low and consists predominantly of macrophages and lymphocytes (Schwarz et al., 2011a,b; Pilla et al., 2012). Nevertheless, recent studies investigating the composition of milk cells in the range $<100,000$ cells $/ \mathrm{mL}$ interestingly revealed evidence for inflammatory reactions based on elevated proportions of PMN (Schwarz et al., 2011a,b; Pilla et al., 2012, 2013). This indicated a correlation between elevated proportions of PMN and IMI or at least an active immune response (i.e., triggered by pathogens). Hence, in addition to SCC, determination of proportions of individual immune cell populations in milk is beneficial for describing the actual udder health status of dairy cows in more (Pillai et al., 2001; Rivas et al., 2001; Pilla et al., 2013). So far, various research groups around the world have developed methods for cell differentiation. However, no routine method is available yet that could be applied in high-throughput milk analyzers that would typically be used in central milk testing (CMT) laboratories.

The objective of this study was to describe and present the development of a new method for simultaneous measurement of a new parameter called differential somatic cell count (DSCC) and SCC.

\section{MATERIALS AND METHODS}

\section{Dairy Farms}

Milk samples used in this study originated mainly from 3 dairy farms. Farms A and B were located in North Zealand, Denmark, whereas farm C was situated in the central part of Jutland, Denmark. In farms A to $\mathrm{C}$, between 120 and 270 dairy cows of the breed Holstein-Frisian were housed in pen barns and milked twice per day in milking parlors, except farm $\mathrm{C}$ which used milking robots (DeLaval, Tumba, Sweden). A TMR mainly consisting of grass and maize silage, rape grist, and cereals was fed on all of the dairy farms. The average herd milk yields ranged between 10,500 and $12,500 \mathrm{~kg} / \mathrm{yr}$ (3.79-3.91\% fat, $3.33-3.41 \%$ protein). The average number of parity and DIM varied from 1.8 to 2.1 and 165 to 198, respectively. Bulk tank milk SCC ranged between 130,000 and 220,000 cells/mL.

\section{Types of Milk Samples Used}

Routinely Available Cow-Composite Samples. Samples used for method development originated from dairy farm A (120 dairy cows) and were received on a weekly basis. Briefly, freshly collected individual cow milk samples, obtained with milk meters (TruTest, Auckland, New Zealand), were available from 20 cows (30 to 200 DIM and parity 1 to 4 ) every Monday morning (d 0). Subsamples of each individual cow milk sample were preserved with Broad Spectrum Micro tabs (Advanced Instruments Inc., Norwood, MA) on d 0 to a final concentration of bronopol and natamycin of 0.27 and $0.01 \mathrm{mg} / \mathrm{mL}$ of milk, respectively. These samples were used for experiments within a maximum period of $4 \mathrm{~d}$.

DHI Samples. Routinely collected DHI samples (bronopol-preserved) from farms A to C were available for various investigations upon request. Samples were available from all lactating animals. These samples were used for different experiments within a maximum period of $4 \mathrm{~d}$ after collection.

Routine DHI Samples. In total, 655 routinely collected bronopol-preserved samples from CMT laboratories in Canada, Denmark, France, and New Zealand were received. Due to the lengthy transportation in some cases, the age of these samples was between 3 and $5 \mathrm{~d}$ on arrival. Samples were processed within $1 \mathrm{~d}$ upon arrival.

Reference Milk Samples. Commercial reference materials were available from Cecalait (Poligny, France), Hüfner (Hergatz, Germany), Max RubnerInstitut (Kiel, Germany), and Eastern Laboratory 
Services Ltd. (Medina, OH). These samples were all provided as preserved raw milk and were not frozen, with the exception of heat-treated and frozen samples from Max Rubner-Institut.

\section{Foss DSCC Method}

Cell Staining. Staining of cells as described herein is based on the Foss DSCC method, which is the subject of the international patent PCT/EP2010/065615 (Holm, 2012). Briefly, Foss DC Dye is diluted 1:10 with Foss DC Diluent to obtain DC Reagent. Milk samples were prepared by inverting the sample cup 10 times, mixing with the DC Reagent (ratio 1:3.2), incubating at $40^{\circ} \mathrm{C}$ for $1 \mathrm{~min}$, followed by brief vortexing and immediate analysis on a flow cytometer.

Flow Cytometric Analysis. Stained samples were analyzed on different types of flow cytometers (for settings used with the different flow cytometers, see Table 1). Development of the method has primarily been conducted using a BD Accuri C6 cytometer (Becton, Dickinson and Company, Franklin Lakes, NJ), but it was also tested on 2 other commercially available flow cytometers. Raw data as well as derived results were compared. The software used for analysis of raw data was CFlow (Accuri) or FlowJo (BD LSR II, and Attune NxT, Thermo Fisher Scientific, Waltham, MA). To obtain the absolute cell count (i.e., SCC), a fixed volume of $50 \mu \mathrm{L}$ of milk was analyzed.

\section{Performance and Validation of the Foss DSCC Method}

Specificity of DSCC. Fluorescence microscopy was used as an internal reference method for cell differentiation, and was applied continuously throughout method development. In total, 180 routinely available cow-composite and DHI samples were analyzed and correlated with DSCC obtained with flow cytometry. Briefly, cells were stained by mixing $100 \mu \mathrm{L}$ of milk with $10 \mu \mathrm{L}$ of DC reagent. Microscopy slides were prepared from $5 \mu \mathrm{L}$ of stained sample and the cells were directly identified by means of size and morphology using a Zeiss Axiostar plus (Carl Zeiss, Oberkochen, Germany) fluorescence microscopy equipped with 40-fold magnification objective and a mercury lamp. In each sample, the first 100 cells observed were differentiated into macrophage, lymphocyte, or PMN. Time deviation between cytometry and microscopy analyses was kept to a minimum. Operator dependence was investigated on freshly prepared microscopy slides from 10 routinely available cow-composite samples. Samples were analyzed in duplicate by 3 operators within $3 \mathrm{~d}$.

Specificity of Total SCC. The Fossomatic FC was used as a reference method for SCC. Samples were preheated for $20 \mathrm{~min}$ at $40^{\circ} \mathrm{C}$, and inverted 10 times before analysis. In general, samples were analyzed with one intake on the Fossomatic FC. The instrument used was regularly maintained and part of an European Unionwide proficiency testing program.

Repeatability. Repeatability of the Foss DSCC method was determined using routinely available cow-composite samples analyzed on a BD Accuri C6 cytometer. In total, 16 samples were analyzed in 5 replicas, and the mean standard deviation for the data set was calculated. Due to the manual gating of cytometer data, operator dependency was considered. Therefore, repeatability between operators gating the same data set, as well as for one operator gating the same data set 3 times, was determined.

Method Robustness. Robustness of the method as such was investigated by testing variations of age of the milk samples, mixing ratio of the reagent, incubation temperature, and time. The effect on obtained dot plots was evaluated regarding separation between cells and milk matrix background. In total, 200 routinely available cow-composite samples were used for these tests.

The Foss DSCC method was further tested toward a range of sample specific factors such as season, region, country, dairy herd management, breed, and so on by analyzing a total number of 655 routine DHI samples originating from Canada, Denmark, France, and New Zealand. Additionally, reference milk samples were tested.

Cell Sorting Trial. Fluorescence-activated cell sorting was used to evaluate the purity of cell populations within the individual groups applying the Foss DSCC method. A total of 12 routinely available cow-composite samples were analyzed using a BD FACSAria II cell sorter and the Foss DSCC method for defining sorting gates. The following settings were

Table 1. Flow cytometers and respective settings used

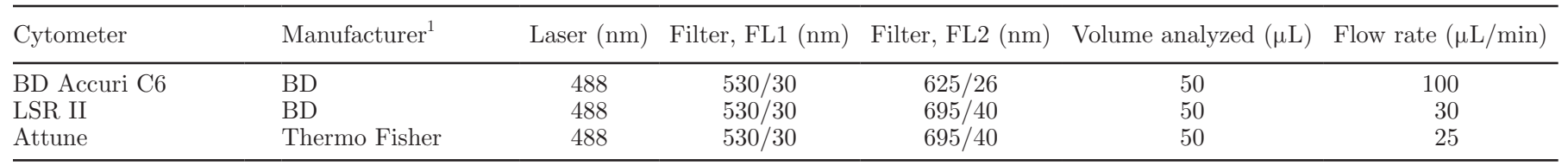

${ }^{1} \mathrm{BD}=$ Becton, Dickinson and Company (Franklin Lakes, NJ); Thermo Fisher Scientific (Waltham, MA). 
used: $488 \mathrm{~nm}$ laser; optical filters FL1-530/30 (FITC) and FL2-695/40 (PerCP); flow rate 4 (corresponding to $4 \mathrm{~mL} / \mathrm{h}$ ); sorting mode: purity; nozzle size $100 \mu \mathrm{m}$. Sorting gates were defined to resemble the 2 groups of cells differentiated by the Foss DSCC method as closely as possible. The 2 sorted fractions were collected in tubes coated with 1\% BSA and kept cold until further analysis. Sorted cells were restained with DC Reagent and were rerun to control survival rate. Cells from the 2 sorted fractions were immediately differentiated using fluorescence microscopy as described above. Samples were only approved if 100 cells could be differentiated in both groups. The whole procedure from collection of milk samples to microscopy was kept within $1 \mathrm{~d}$ to ensure cell viability.

Investigation of Relation to Other Methods for Cell Differentiation. To check the relation between the Foss DSCC method and other methods for cell differentiation described in the literature, 35 routinely available cow-composite samples were used. All samples were analyzed with 4 different methods for cell differentiation: (1) Foss DSCC method (flow cytometer), (2) Foss fluorescence microscopy, (3) flow cytometry after antibody staining (Schwarz et al., 2011a), and (4) light microscopy (Schwarz et al., 2011b). Briefly, the 70-mL cow-composite samples were thoroughly stirred and then subdivided into 3 subsamples consisting of $10 \mathrm{~mL}$ each for methods 1 and 2, $40 \mathrm{~mL}$ for method 3, and 10 $\mathrm{mL}$ for method 4. Samples used for methods 1 and 2 were preserved with bronopol, as described previously. For methods 3 and 4 , cells were isolated from milk in 2 washing steps $\left(15 \mathrm{~min}\right.$ at $200 \times g$ and $\left.4^{\circ} \mathrm{C}\right)$. The washed pellet was stained with volumes of 0.5 and $1 \mu \mathrm{L}$ of primary antibodies against CD11b and CD14 molecules (MM10A and MM61A, Washington State University Monoclonal Antibody Center, Pullman, WA), respectively, for identification of macrophages and PMN according to Schwarz et al. (2011a). In a second step, volumes of each $1 \mu \mathrm{L}$ of secondary antibodies against anti-CD11b and anti-CD14 with fluorescence markers (order no. 553395 and 340270, Becton, Dickinson and Company) were applied. Stained samples were analyzed with a BD LSRII flow cytometer (Becton, Dickinson

Table 2. Descriptive statistical results of the distribution of cell types in routinely available cow-composite and DHI samples $(\mathrm{n}=293)$ analyzed with a fluorescence microscope ${ }^{1}$

\begin{tabular}{lrrcc}
\hline Cell population (\%) & Mean & \multicolumn{1}{c}{ SD } & Minimum & Maximum \\
\hline Lymphocytes & 5.09 & 4.05 & 0 & 19 \\
Macrophages & 35.45 & 14.30 & 7 & 79 \\
PMN & 58.68 & 16.19 & 13 & 92 \\
\hline
\end{tabular}

${ }^{1}$ Somatic cell counts of the analyzed samples ranged between 20,000 and 4,218,000 cells/mL with a mean of 199,807 cells $/ \mathrm{mL}$. and Company) and the FACS Diva software applying the same criteria as described by Schwarz et al. (2011a). In case of method 4, the cell pellet was transferred to a microscope slide, stained and examined according to Schwarz et al. (2011b). As for methods 2, 3, and 4 lymphocyte and PMN proportions were determined individually but then combined to DSCC for further analyses.

\section{Data Analysis}

Analysis and visualization of the data were done with Microsoft Office Excel 2007 (Microsoft Corporation, Redmond, WA). Pearson correlation coefficients (r) were calculated using Matlab R2016a (The MathWorks Inc., Natick, MA).

\section{RESULTS}

\section{Differentiation of Cells in Milk Using Fluorescence Microscopy}

The distribution of the cell types lymphocytes, macrophages, and PMN in a representative set of 293 routinely available cow-composite samples was investigated using fluorescence microscopy. Lymphocytes were generally found with low proportions (mean \pm SD: 5.09 $\pm 4.05 \%$, Table 2). Whereas PMN occurred with the highest average proportion $(58.68 \pm 16.19 \%)$, mean values for macrophages were $35.45 \pm 14.30 \%$. The SCC values of the samples analyzed ranged between 20,000 and 4,218,000 cells $/ \mathrm{mL}$.

Proportions of lymphocytes varied between 0 and $19 \%$ across the entire SCC range analyzed (Figure 1) and did not indicate any correlation to SCC ( $\mathrm{r}=$ $-0.1672, P<0.01)$. Macrophages varied in a wide range from 7 to $79 \%$ but indicated decreasing proportions as SCC increased $(\mathrm{r}=-0.3400, P<0.001)$. The PMN were found with high variations, from 13 to $92 \%$, and a trend of higher proportions as SCC increased ( $\mathrm{r}$ $=0.3604, P<0.001$ ).

\section{Definition of the DSCC Parameter}

Differential SCC is a new parameter for mastitis screening and represents the combined proportion of the 2 milk cell populations PMN and lymphocytes expressed in percentage. The proportion of macrophages can be calculated by subtracting DSCC from $100 \%$. The combination of cell staining, flow cytometry technology, and use of individual cell properties enables SCC and DSCC determination using the Foss DSCC method (Figure 2). Specifically, fluorescence emission signals from channels 1 and 2 are used for determina- 

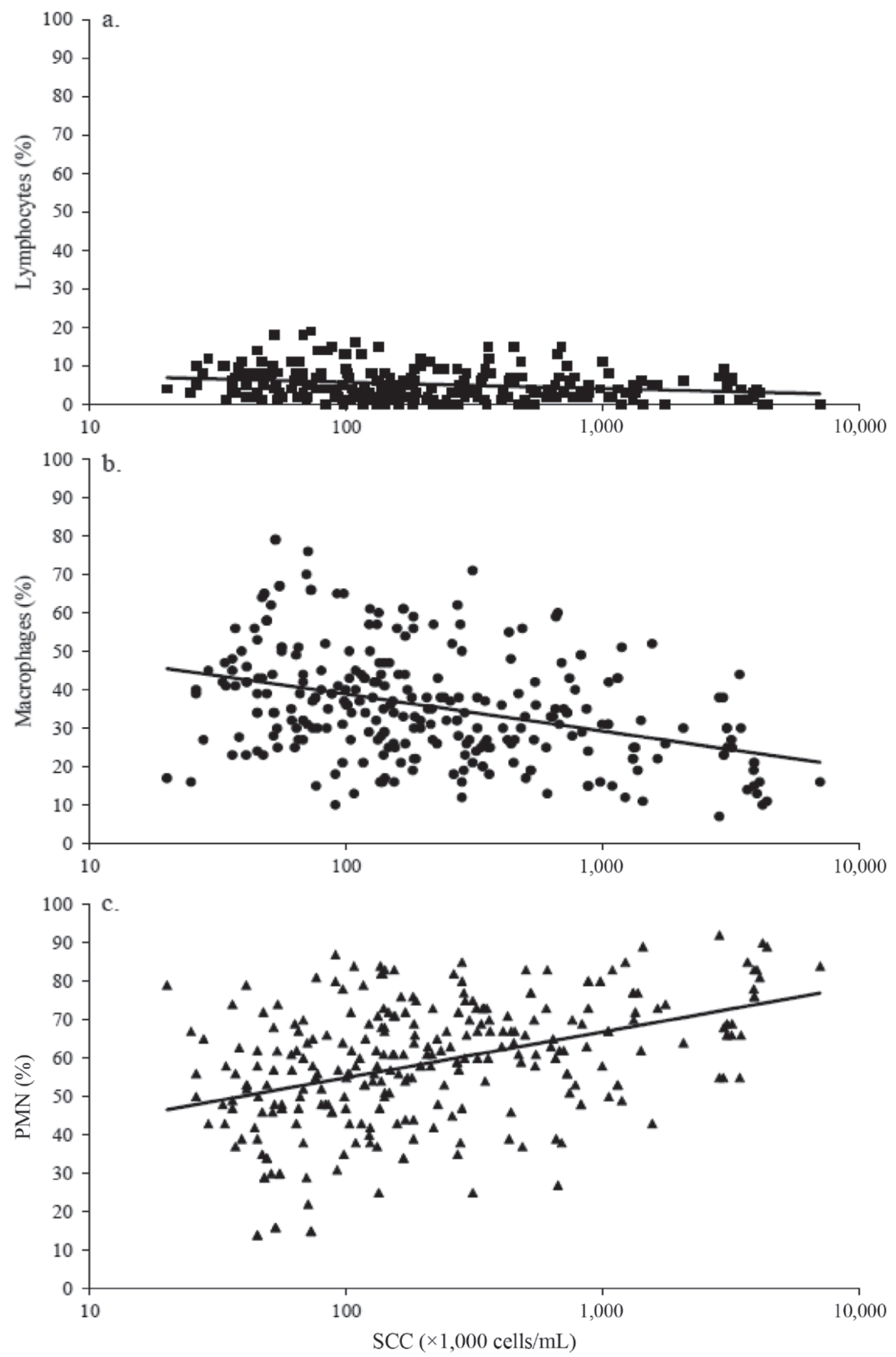

Figure 1. Proportions of (a) lymphocytes, (b) macrophages, and (c) PMN determined by the Foss differential somatic cell count (DSCC) method applied on a fluorescence microscope depending on SCC. In total, 293 routinely available cow-composite and DHI samples were analyzed. Data are illustrated in combination with logarithmic trendlines. Each symbol represents the result of one cow-composite sample, but overlapping is possible. 
Table 3. Descriptive statistics for differential somatic cell count (DSCC) values (\%) obtained on 3 different flow cytometers using 2 different data sets of routinely available cow-composite samples

\begin{tabular}{lccccc}
\hline Flow cytometer $^{1}$ & $\mathrm{n}$ & Mean & SD & Minimum & Maximum \\
\hline BD Accuri C6 & 40 & 56.61 & 10.48 & 38 & 77 \\
Attune NxT & 40 & 59.34 & 9.47 & 40 & 79 \\
BD Accuri C6 & 38 & 68.85 & 9.51 & 47 & 83 \\
BD LSRII & 38 & 69.91 & 9.71 & 43 & 81 \\
\hline
\end{tabular}

${ }^{1}$ BD Accuri C6 and BD LSRII, Becton, Dickinson and Company (Franklin Lakes, NJ); Attune NxT, Thermo Fisher Scientific (Waltham, MA).

tion of the SCC (Figure 2A). In a second step, only cells (i.e., SCC) identified in Figure 2A are further investigated in a dot plot at higher magnification (Figure 2B). This allows differentiation between macrophages and PMN together with lymphocytes appearing in a separate group.

Routinely available cow-composite samples were used to investigate DSCC values determined using 3 different types of flow cytometers (Table 3 ). The SCC values of the samples analyzed ranged between 30,000 and 1,411,000 cells $/ \mathrm{mL}$. The DSCC results obtained from BD Accuri C6 and Attune NxT correlated highly ( $\mathrm{r}=$ $0.9679, P<0.001)$. A similarly high correlation of $\mathrm{r}=$ $0.9794(P<0.001)$ was calculated comparing results from BD Accuri C6 and BD LSRII flow cytometers.

\section{SCC}

The Foss DSCC method provides both DSCC and SCC in one analysis. In total, 996 routinely available cow-composite samples as well as DHI samples were used for investigation of the relation between SCC obtained with the new Foss DSCC method on a commercially flow cytometer and the Fossomatic FC. The SCC values in the samples analyzed ranged between 3,000 and 3,743,000 cells/mL (Figure 3) and correlated highly significantly $(\mathrm{r}=0.9950, P<0.001)$.

\section{Performance and Validation of the Foss DSCC Method}

Specificity. Fluorescence microscopy was used as an internal reference method. The DSCC values obtained using the Foss DSCC method were compared with those obtained by fluorescence microscopy (Figure 4). The data set represented 180 samples with SCC ranging from 50,000 to 1,500,000 cells $/ \mathrm{mL}$, and DSCC ranging from 43 to $93 \%$. A high correlation of $\mathrm{r}=0.8456(P<$ 0.001) was calculated.

Operator dependency was investigated by analysis of 10 routinely available cow-composite samples in duplicates (Table 4). Notably, the DSCC (lymphocytes
+ PMN) followed the same pattern for microscopy and cytometer analysis, and represented a wide range from 37 to $95 \%$. The number of lymphocytes found by each operator was relatively low and constant between
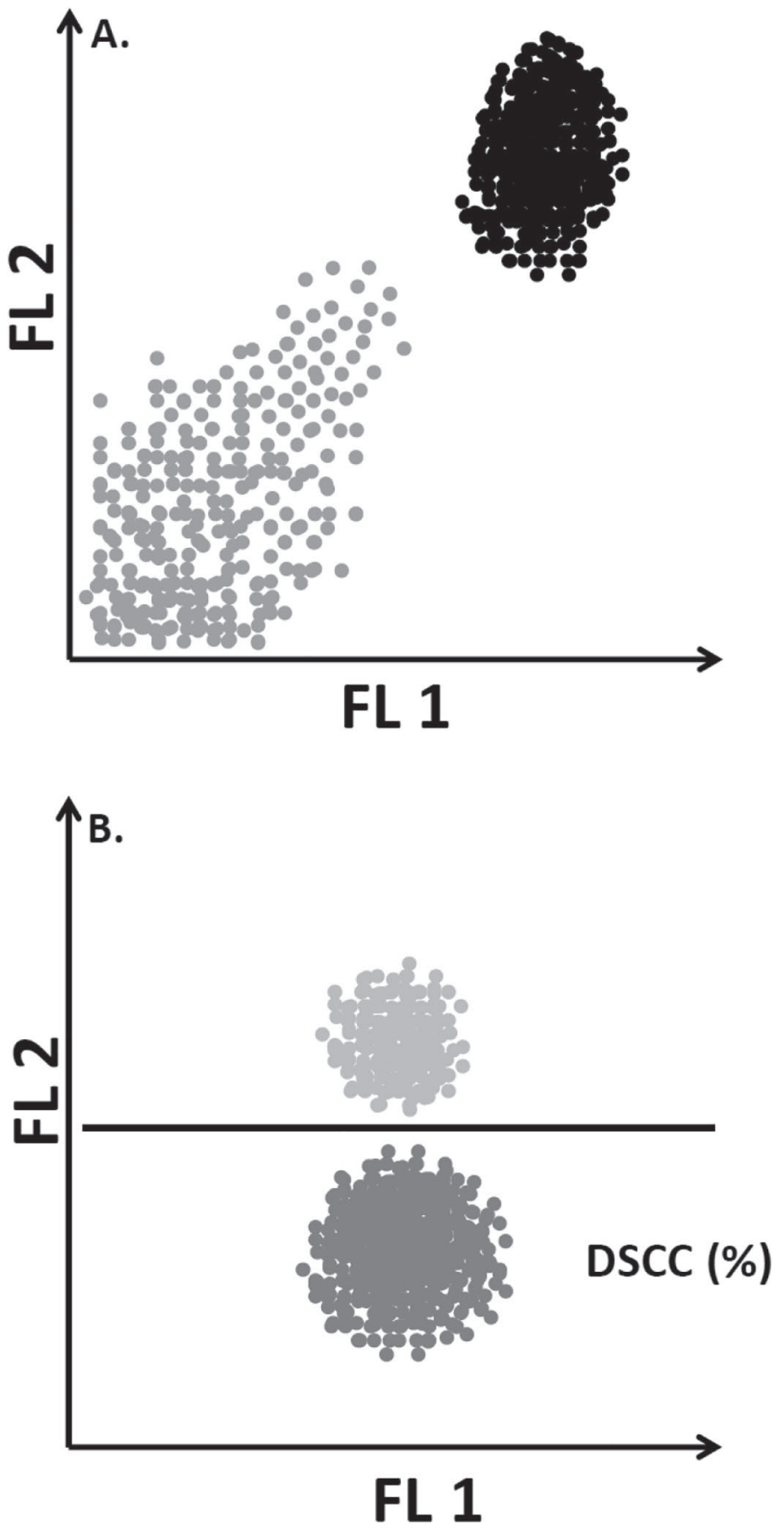

Figure 2. Schematic diagram of the counting principle. (A) Separation between background (gray dots) and milk cells (black dots) fluorescence information and determination of SCC in cells per milliliter. (B) Only somatic cells from A are used for determination of differential somatic cell count (DSCC) by differentiating macrophages (light gray dots) from lymphocytes and PMN (dark gray dots). FL = fluorescence channel. 
samples. Overall, the repeatability of one operator analyzing DSCC in the same sample twice was high (SD of $2.8 \%$ ).

Examples of the 3 different cell types identified with fluorescence microscopy are illustrated in Figure 5 . Macrophages were identified as being the largest cell type, the cytoplasm constituting the larger part of the cell as compared with the nucleus. Besides the nucleus, cell granules were clearly stained. The PMN were identified as being in between macrophages and lymphocytes in size, with the characteristic of a distinctive subdivided nucleus. Lymphocytes were the smallest cell type, with almost no visible cytoplasm surrounding the fully stained nucleus.

Repeatability. The method repeatability on the $\mathrm{BD}$ Accuri C6 cytometer was determined by analysis of 16 routinely available cow-composite samples, run in 5 replicas, and found to be good (SD 2\%). The data set consisted of samples with SCC ranging from 30,000 to
850,000 cells/mL and DSCC ranging from 33 to $96 \%$. When assessed separately, the SD for one operator gating the raw data was $1.3 \%$, and the SD between operators was found to be $2.7 \%$.

Robustness. Method-specific robustness was tested toward sample age, mixing ratio between milk and DC Reagent, incubation temperature, and incubation time (Table 5). These factors were tested on 200 routinely available cow-composite samples, with SCC typically ranging from 50,000 to 1,500,000 cells/mL and DSCC from 40 to $90 \%$. Tests of the mixing ratio between milk and DC Reagent showed a reduction of both cell type information and separation between background and cells when the amount of reagent was reduced in relation to milk. The effect of incubation temperature was tested in the range from 35 to $45^{\circ} \mathrm{C}$, with an attenuation of background/cell signal separation at the lower temperature. A similar effect was observed in general for incubation times shorter than $60 \mathrm{~s}$; after $30 \mathrm{~s}$ of in-

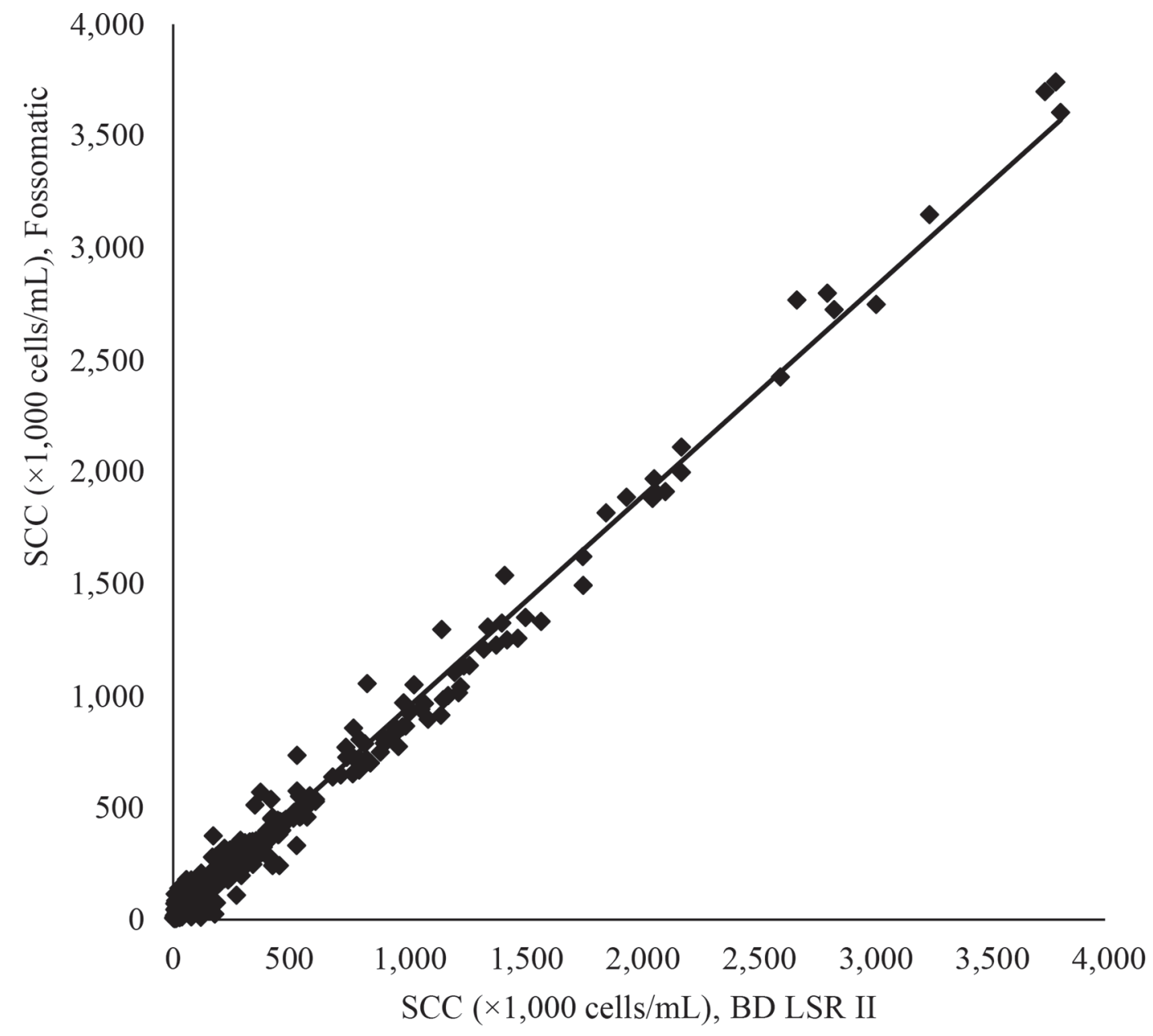

Figure 3. Somatic cell count results of $n=996$ routinely available cow-composite and DHI samples analyzed on the Fossomatic FC (Foss Analytical A/S, Hillerød, Denmark) and, with the new Foss differential somatic cell count (DSCC) method, on a BD LSR II instrument (Becton, Dickinson and Company, Franklin Lakes, NJ). 


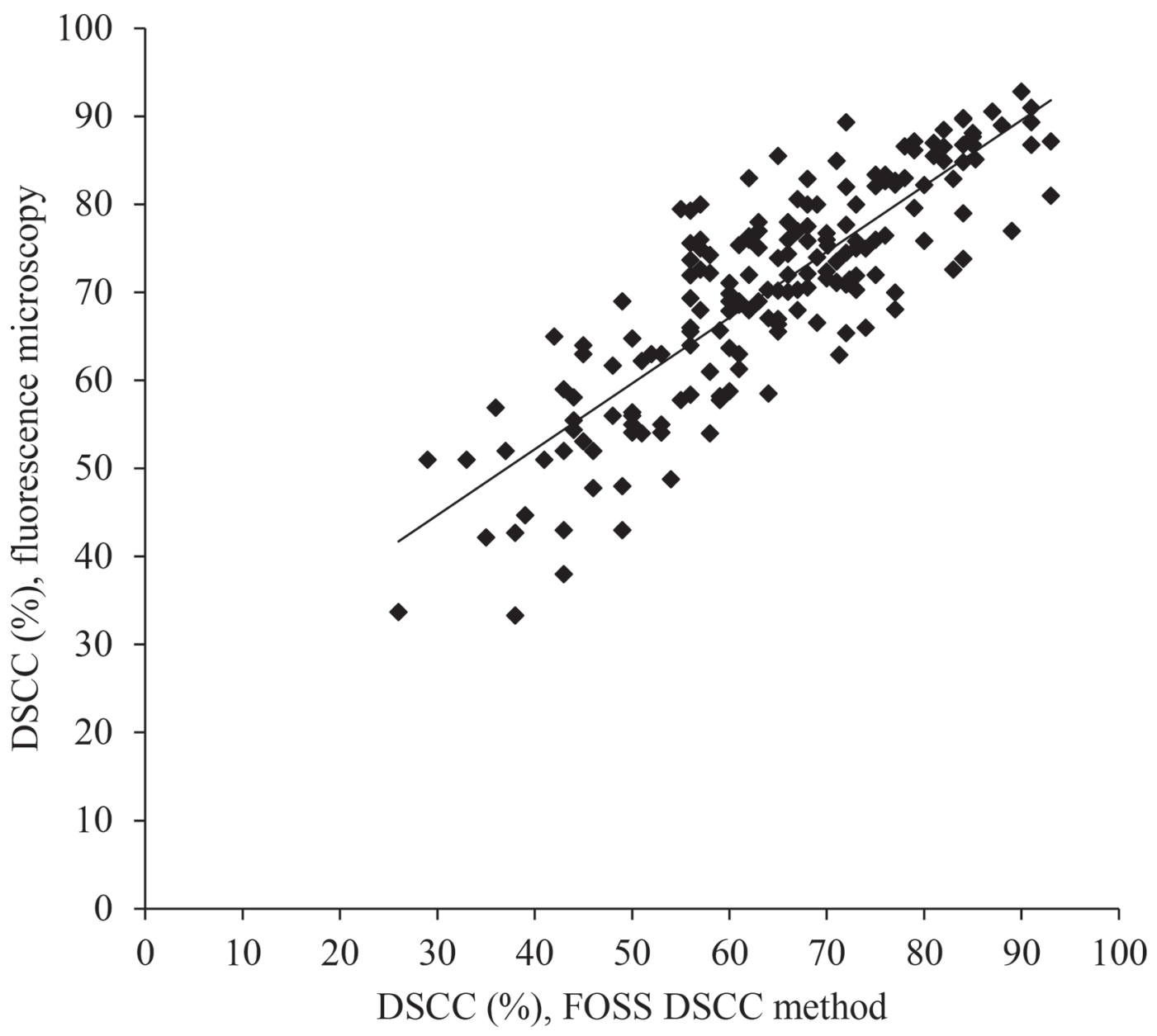

Figure 4. Specificity of the Foss differential somatic cell count (DSCC) method shown as correlation between differential somatic cell count (DSCC) percentage obtained with a BD Accuri C6 cytometer (Becton, Dickinson and Company, Franklin Lakes, NJ) and with fluorescence microscopy $\left(\mathrm{R}^{2}=0.7\right)$. Data are shown for 180 routinely available cow-composite samples, with SCC between 25,000 and $2,500,000$ cells $/ \mathrm{mL}$.

cubation, staining of cells was less complete than when the incubation process was allowed to proceed for a longer time. In general, tested conditions were evaluated based on separation between background signal and cell signal; a condition resulting in a lower signal to noise ratio (e.g., less reagent relative to milk) may still give comparable DSCC and SCC for some samples but might render the method less robust in general.

The DSCC method was developed for DHI sample analysis and should therefore be robust toward a range of factors that could affect it. Thus, 655 routine DHI samples representing a broad variation of samplespecific factors such as season, region, country, dairy herd management, breed, and so on were considered in the development of the Foss DSCC method. The DSCC and SCC values were determined for all of the samples received without any difficulty (Table 6). Mean values for DSCC ranged between 72.68 (Denmark) and $76.12 \%$ (Canada). Overall, DSCC values varied in simi- lar ranges across all countries with the lowest values occurring in Canada and New Zealand.

Currently, reference milk samples with defined SCC are used to ensure stable performance of SCC analysis in CMT laboratories globally. Because the Foss DSCC method was developed to provide both DSCC and SCC in DHI samples, the possibility of analyzing such reference samples was assessed. All SCC test results were within the SCC ranges stated by the suppliers of the reference milk samples. It was not possible to measure DSCC in the reference milk samples.

Cell Sorting Trial. Grouping of cells according to the Foss DSCC method was investigated in a cell sorting experiment followed by fluorescence microscopy of sorted cells. Restaining and reanalysis of sorted cells showed a survival rate of about $50 \%$ (data not shown). The share of intact milk cells after sorting was optimized by performing the whole process (i.e., from milking to fluorescence microscopy) within $12 \mathrm{~h}$. 


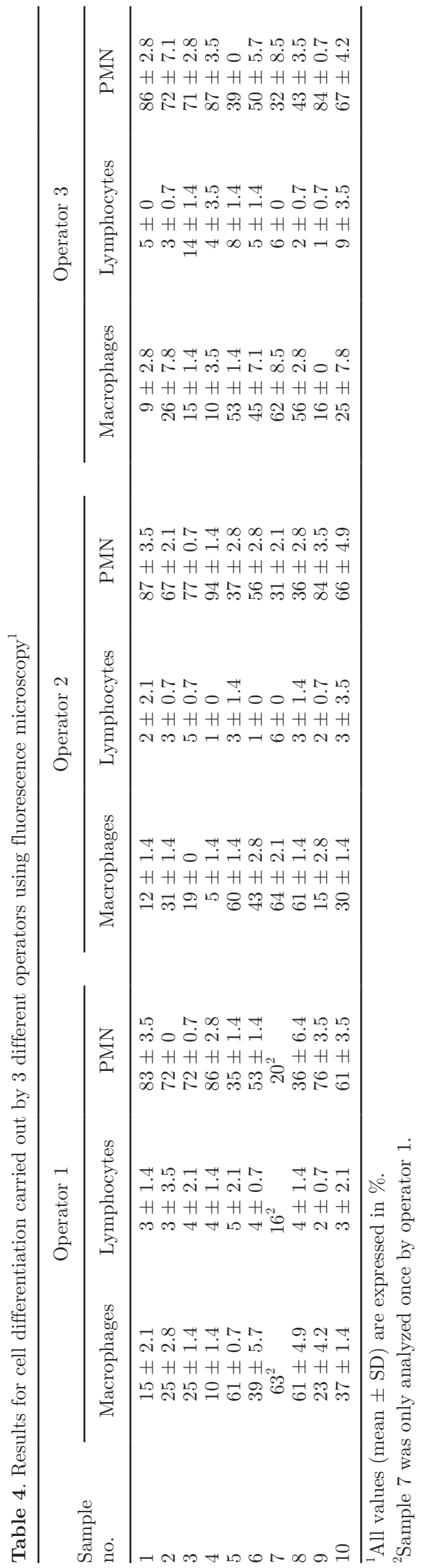

In total, 12 milk samples with SCC ranging between 90,000 and 3,000,000 cells/mL were analyzed (Table 7). In general, comparable high purities were found across all samples analyzed with an average of $94 \%$ PMN + lymphocytes and $83 \%$ macrophages in fractions DSCC and $100 \%$ minus DSCC, respectively.

Relation to Other Methods for Cell Differentiation. To evaluate the relation between the new Foss DSCC method and existing methods for cell differentiation, a total of 4 different methods for cell differentiation as described above were applied on a set of 35 routinely available cow-composite samples.

The SCC in the samples analyzed ranged between 20,000 and 832,000 cells $/ \mathrm{mL}(150,450 \pm 164,500$ cells/ $\mathrm{mL})$. The DSCC determined with method 1 varied between 43 and $87 \%$ (66.78 $\pm 9.99 \%$; Figure 6). Applying method 2, DSCC values from 43 to $90 \%$ (66.13 $\pm 12.16 \%$; Figure 6a) were observed. A correlation coefficient of $\mathrm{r}=0.8051(P<0.001)$ between these 2 methods was found. The DSCC determined with method 3 showed variations between 56 and $90 \%$ (74.11 $\pm 8.81 \%$; Figure $6 \mathrm{~b}$ ). A correlation between methods 1 and 3 could not be found $(\mathrm{r}=0.3361, P=0.0558)$. The DSCC values measured with method 4 were in a broad range between 26 and 90\% (55.97 $\pm 15.29 \%$; Figure $6 \mathrm{c})$. Methods 1 and 4 did not correlate with each other $(\mathrm{r}=0.3520, P=0.0532)$.

\section{Initial Field Trial}

The Foss DSCC method is sought for providing more information in terms of mastitis management through regular DHI testing programs. In an initial field trial, in total 190 DHI samples originating from dairy farm B were analyzed.

The SCC ranged between 14,000 and 1,119,000 cells/ $\mathrm{mL}(202,030 \pm 232,970$ cells $/ \mathrm{mL}$; Figure 7$)$. The DSCC occurred in a broad range from 34 to $87 \%$ (63.12 \pm $9.06 \%)$.

High proportions of 66 and $89 \%$ of the samples tested appeared in the ranges of $<200,000$ and $<400,000$ cells/
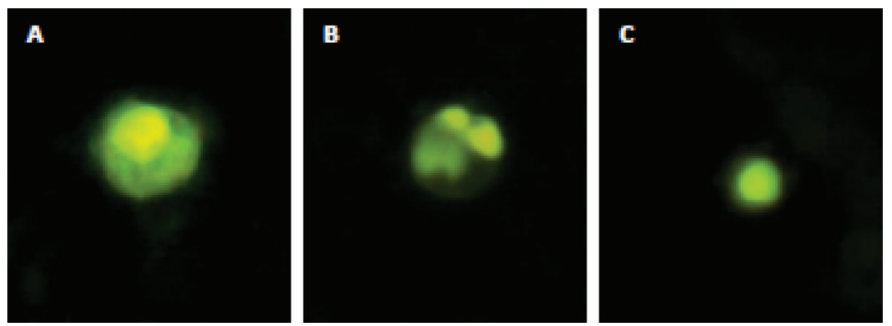

Figure 5. Representative examples of macrophage (A), PMN (B), and lymphocyte (C) are shown (40-fold magnification) analyzed using fluorescence microscopy. Color version available online. 
Table 5. Method robustness regarding separation between background and cells

\begin{tabular}{llll}
\hline Parameter & \multicolumn{1}{c}{ Range tested } & SOP ${ }^{1}$ conditions & Effect on robustness \\
\hline Sample age (d) & $1-5^{2}$ & $1-4$ & Reduction after $>4 \mathrm{~d}$ \\
& $0-5^{3}$ & $1-3$ & Reduction after $>3 \mathrm{~d}$ \\
Mixing ratio & $\pm 10 \%$ (vol) DC Reagent ${ }^{4}$ & $1: 3.2$ (milk:DC Reagent) & $\begin{array}{l}\text { Reduction with less reagent than SOP; }+10 \% \text { reagent } \\
\text { has no apparent effect }\end{array}$ \\
Incubation temperature $\left({ }^{\circ} \mathrm{C}\right)$ & $35-45$ & Reduction at $35^{\circ} \mathrm{C} ;$ no apparent effect at $45^{\circ} \mathrm{C}$ \\
Incubation time (s) & $30-90^{5}$ & 60 & Reduction with $<60$ s; no apparent effect of $>60 \mathrm{~s}$ \\
\hline
\end{tabular}

${ }^{1} \mathrm{SOP}=$ standard operating procedure.

${ }^{2}$ Preserved samples stored at room temperature.

${ }^{3}$ Unpreserved samples stored at $5^{\circ} \mathrm{C}$. Day 0 means on the day of sample collection.

${ }^{4}$ Foss DC Dye (Foss Analytical A/S, Hillerød, Denmark) is diluted 1:10 with Foss DC Diluent to obtain DC Reagent.

${ }^{5}$ The method is run manually without autosampler and hence incubation times are not totally precise.

$\mathrm{mL}$, respectively. A minor proportion of $11 \%$ revealed values $>400,000$ cells $/ \mathrm{mL}$. The DSCC values varied broadly from 34 to $79 \%$ in samples with $<400,000$ cells $/ \mathrm{mL}$ (Figure 7 ). The variation of DSCC values in samples with $>400,000$ cells $/ \mathrm{mL}$ was in a narrow band from 53 to $89 \%$ and at a higher level. The DSCC values tended to increase as SCC values increased. However, the correlation between SCC and DSCC was weak $(\mathrm{r}=$ $0.3852, P<0.001)$.

\section{DISCUSSION}

Numerous indicators for mastitis (e.g., $N$-acetyl$\beta$-glucosaminidase activity, electrical conductivity) have been developed and investigated over the last half century (Kitchen, 1981; Pyörälä, 2003). However, SCC, typically determined on highly accurate highthroughput analyzers became the key parameter in routine mastitis monitoring programs in the frame of DHI testing since its introduction in the early 1980s. Despite extensive mastitis control programs being in place for several decades, mastitis remains one of the most significant diseases affecting dairy cows worldwide, resulting in large economic losses to the dairy industry (e.g., Halasa et al., 2007). Hence, more sensitive and inexpensive biomarkers are still needed (Viguier et al., 2009). Several studies (Rivas et al., 2001; Pillai et al., 2001; Schwarz et al., 2011a,b; Pilla et al., 2012, 2013) revealed merit in differentiating milk immune cells in addition to determining the total SCC for a more definite description of the udder health status of dairy cows. The aim of this work was to develop a new method for routine mastitis screening based on the simultaneous analysis of a new parameter, DSCC, and the well-established SCC in individual cow milk samples (i.e., DHI samples).

\section{New Foss DSCC Method}

In assessing the requirements on the new method for simultaneous determination of DSCC and SCC, accuracy, reliability, repeatability, robustness, low cost, and high-throughput became top priority. The Foss DSCC method was developed on bench-top flow cytometers, but is aimed to be applied on dedicated flow cytometry-based high-throughput analyzers in CMT laboratories. Time and cost-intensive sample preparation (e.g., dilution, centrifugation, or both) clearly had to be kept to a minimum to match the speed of current Fossomatic instruments, where up to 600 milk samples can be analyzed per hour.

Counting and differentiation of somatic cells using the new Foss DSCC method is based on fluorescent staining of cell nuclei and other compartments (e.g., granula) applied on individual cow milk samples. In this context, well-known cell characteristics and morphology (e.g., Lee et al., 1980) were used. In the absence of a reference method or gold standard for milk cell differentiation, an internal fluorescence microscopy method, identifying cells by morphology and size, was

Table 6. Descriptive statistics for differential somatic cell count (DSCC) and SCC measured in routine DHI samples

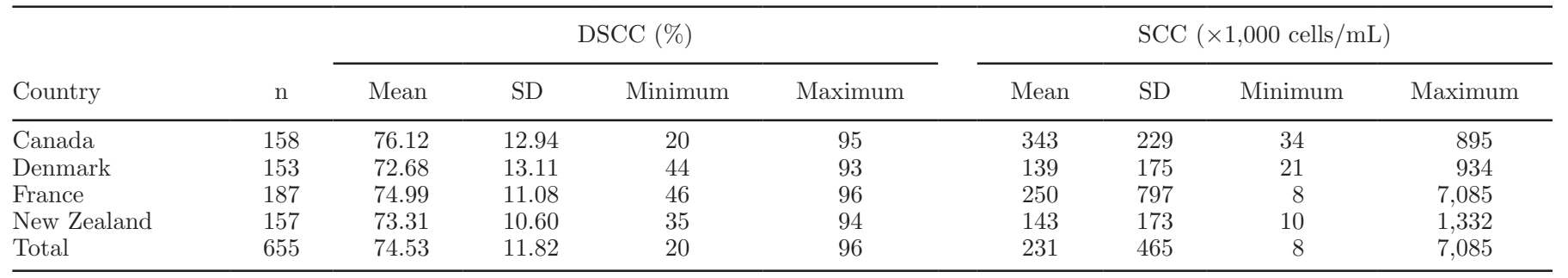


Table 7. Purity of cells in the fractions differential somatic cell count (DSCC) and $100 \%$ minus DSCC analyzed by fluorescence microscopy after cell sorting on a BD FACSAria $\mathrm{II}^{\mathrm{I}}$

\begin{tabular}{lccc}
\hline Sample no. & SCC $(\times 1,000$ cells $/ \mathrm{mL})$ & Purity $(\%)$, DSCC & Purity $(\%), 100 \%$ DSCC \\
\hline 1 & 92 & 67 & 94 \\
2 & 1,000 & 81 & 93 \\
3 & 3,000 & 78 & 94 \\
4 & 178 & 84 & 95 \\
5 & 630 & 85 & 93 \\
6 & 971 & 88 & 96 \\
7 & 455 & 87 & 90 \\
8 & 440 & 88 & 97 \\
9 & 870 & 84 & 93 \\
10 & 410 & 87 & 92 \\
11 & 1,600 & 86 & 95 \\
12 & 303 & $83.08 \pm 5.90$ & $93.67 \pm 1.92$ \\
All (mean \pm SD) & $829.08 \pm 803.90$ & &
\end{tabular}

${ }^{1}$ Becton, Dickinson and Company (Franklin Lakes, NJ).

developed to investigate the specificity of the Foss DSCC method. Although microscopy is often associated with drawbacks such as time consumption and operator dependency (Schröder and Hamann, 2005), it was our method of choice for verification of the new flow cytometric method. Sample preparation for microscopy was kept the same as for flow cytometry to reduce variations in cell type distribution due to methodology to a minimum. The high correlation coefficient of $\mathrm{r}=$ 0.8456 found between the 2 methods illustrated that cells can be differentiated in a highly specific manner using the Foss DSCC method. However, the accuracy of flow cytometry is generally described to be superior compared with microscopy given that higher numbers of cells are analyzed (e.g., Koess and Hamann, 2008).

The investigation of the relation between the Foss DSCC method and microscopy as well as flow cytometry methods described in literature (Schwarz et al., 2011a,b) revealed that results for individual cell populations obtained using different methods did not correlate with each other. This observation might be explainable by the fact that with literature methods cells need to be isolated from the milk matrix, whereas untreated milk samples were used with the Foss DSCC method. It is well known (Schröder and Hamann, 2005; Li et al., 2015 ) that centrifugation and washing of cells can alter the distribution of cell types occurring in milk.

The specificity of the Foss DSCC method was further investigated in a cell sorting experiment, where cells originating from the 2 different gates used for differentiation were sorted and subsequently analyzed using fluorescence microscopy. Fluorescence-activated cell sorting is generally accepted as a suitable technique to sort cells with high purities of 95 to $100 \%$ out of the respective gates (Basu et al., 2010). Our experiments revealed high purities of 83 and $94 \%$ of the respective cell populations differentiated by the Foss DSCC method. This result was interpreted as another confirmation of the specificity of our new method in terms of differentiation of cells. Beyond that, the accurate correlation of SCC measured on an existing Fossomatic FC instrument with those generated using the Foss DSCC method confirmed that all cells occurring in milk were stained and thus included in the determination of DSCC.

The simultaneous determination of DSCC and SCC implies that a defined volume of $50 \mu \mathrm{L}$ of milk is analyzed to be able to calculate the number of cells per milliliter. Hence, the number of cells available to determine DSCC clearly depends on SCC. To obtain sufficient statistics for the output, both DSCC and SCC, the performance range for the method was defined to be 50,000 to $1,500,000$ cells $/ \mathrm{mL}$.

The Foss DSCC method was specifically developed for individual cow milk (DHI) samples, which are typically preserved. Bronopol was used as the standard preservative during method development. However, reduced stability of the cell type information was observed for a smaller set of samples preserved with azidiol (data not shown). The reason for this might be a too harsh effect of azidiol on the cellular compartments (Barcina et al., 1987), resulting in altered cell type information. Nevertheless, the nuclei seemingly remained intact and thus reliable generation of SCC values in samples containing azidiol was possible.

Method robustness was tested regarding the quality of raw data as the separation between background signal from stained milk matrix and cell signal, and also on the stability of the cell type information. Increased sample age influenced quality of the result by reducing both the number and quality of the milk cells, which is in accordance with observations of others (e.g., $\mathrm{Li}$ et al., 2015). Cells that were dying or decaying cells might still be stained, but this would result in a lower mean fluorescence and hence in a broadening of the cell population signal. Higher sample age also means a risk 

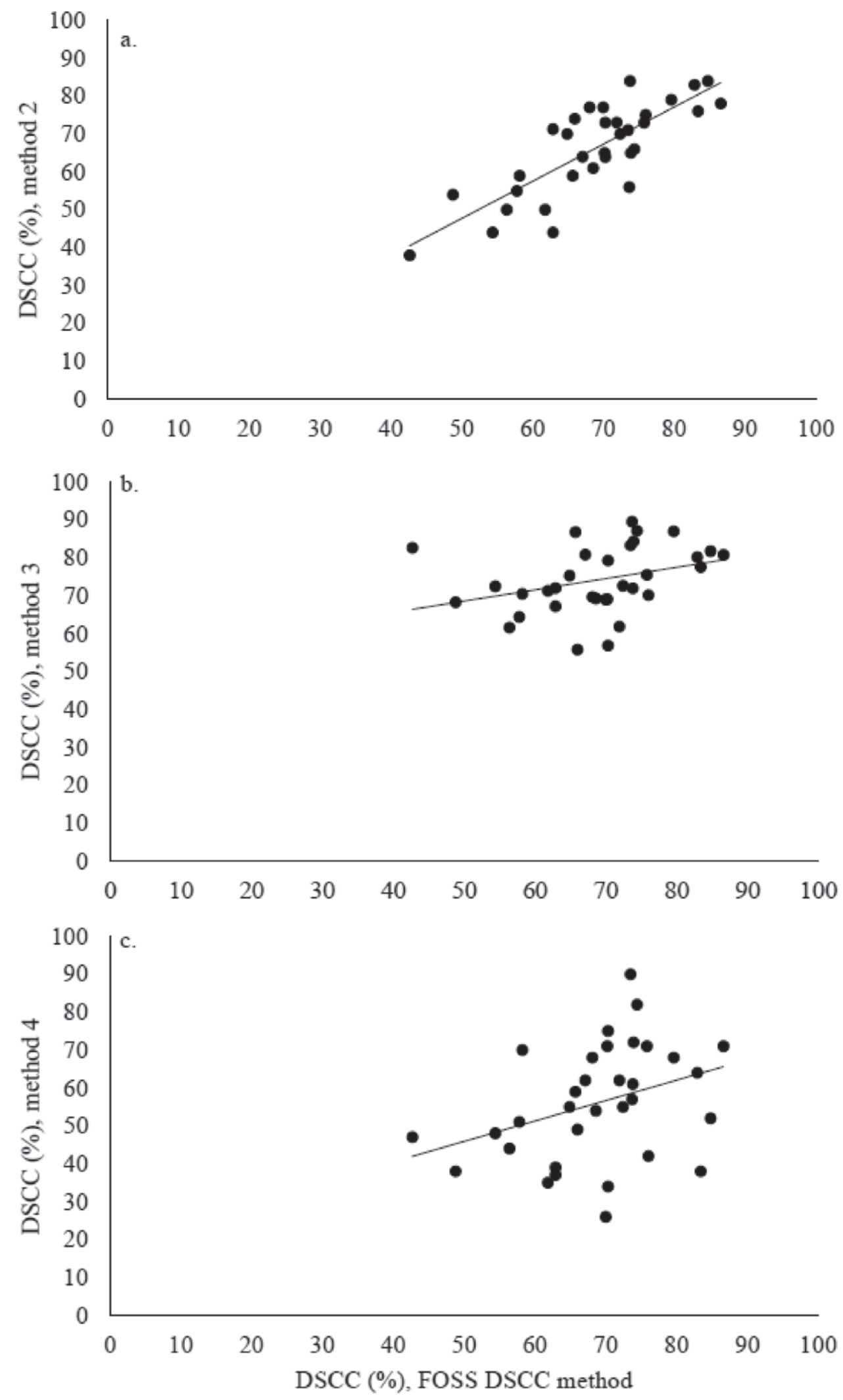

Figure 6. Results of the investigation of the relation between different methods for cell differentiation: (1) Foss differential somatic cell count (DSCC) method, (2) fluorescence microscopy, (3) flow cytometry after antibody-staining, and (4) light microscopy. Data are illustrated in combination with linear trendlines. Each symbol represents the result of one cow-composite sample, but overlapping is possible. 


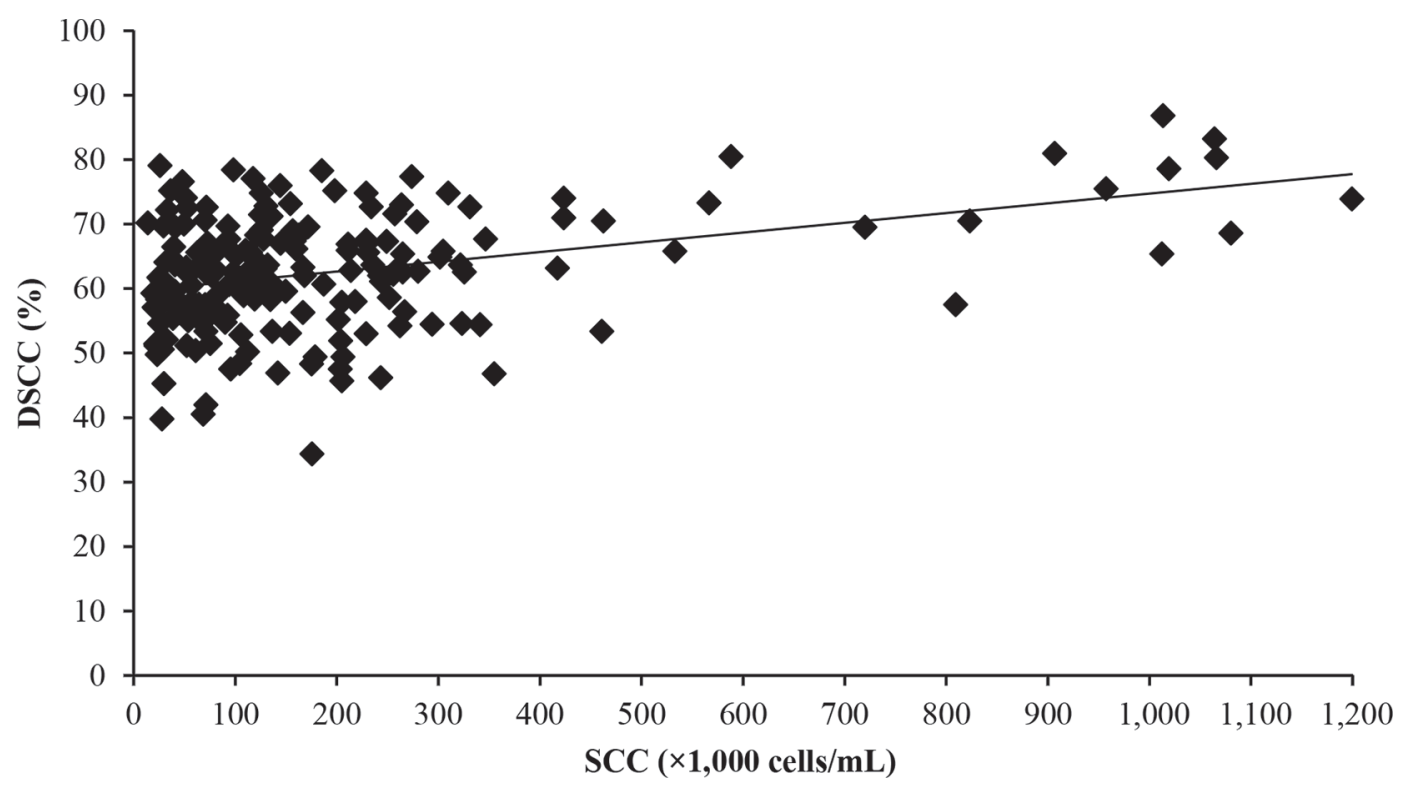

Figure 7. Differential somatic cell count (DSCC) versus SCC plot for results of initial field study using DHI samples from one dairy farm $(\mathrm{n}=188)$. Data are illustrated in combination with a linear trendline. Each symbol represents the result of one cow-composite sample, but overlapping is possible.

of bacterial growth, depending on sample storage and preservation. The combined effect might result in worse separation between background and cells. Hence, for a reliable generation of DSCC values, the maximum age of milk samples should not exceed $5 \mathrm{~d}$. The decrease in separation observed at lower incubation temperature was probably due to a combination of a lower degree of cell staining and more interference of stained fat droplets at temperatures lower than $40^{\circ} \mathrm{C}$. The importance of the incubation time was also tested, and a reduced separation between background and cells was observed at incubations shorter than $60 \mathrm{~s}$.

The method development was mainly based on metered individual cow milk samples obtained from a local dairy farm on a weekly basis. Beyond that, the robustness of the method toward different factors such as season, region, country, dairy herd management, breed, and so on was tested by analyzing milk samples that originated from various locations around the globe. Given that both proper DSCC and SCC values could be generated under different conditions using the Foss DSCC method, we assume that the Foss DSCC method is applicable in different DHI testing environments.

\section{New DSCC Parameter}

Differential somatic cell count was defined as the combined proportion of PMN and lymphocytes expressed in percentage and is sought to be a new parameter for mastitis screening that can be applied in the frame of DHI testing. Macrophages are differentiated in a separate group and proportions can be calculated by subtracting DSCC from 100\%. Lymphocytes and PMN were combined into one group because proportions of lymphocytes appeared to be constantly low across the entire SCC range in all of our studies. Instead, an antidromic trend of the development of macrophages and PMN as SCC increased was observed.

Recent cell differentiation studies (Schwarz et al., 2011a,b; Pilla et al., 2012, 2013) described proportions of macrophages to be fairly constant, whereas percentage of lymphocytes decreased while the percentage of PMN increased as SCC increased. The different results might be explainable by the fact that evidently different methods for cell differentiation were applied. Whereas our method is based on the analysis of untreated individual cow milk samples, cell differentiation in literature studies was generally performed after various centrifugation and washing steps, which can alter the composition of cells as discussed above.

Moreover, the type of milk samples analyzed was evidently different. Our studies were based on the analysis of metered individual cow milk samples, whereas quarter foremilk samples were used in literature studies. Both total SCC as well as the composition of cells vary during the milking procedure (Sarikaya et al., 2005). The SCC values were higher in cisternal compared with alveolar milk and increased significantly in residual milk. Macrophages were found as predominant cell population in cisternal milk. However, PMN dominated in all remaining milk fractions. 
Nevertheless, the increase in proportions of PMN as SCC increased determined by the Foss DSCC method was in accordance with previously published studies (Paape et al., 1979; Kehrli and Shuster, 1994; Rivas et al., 2001; Schwarz et al., 2011a,b) and equally high proportions of up to $90 \%$ PMN were also found. In this context, the definition of DSCC as the group of cells containing PMN aimed to follow the cell type alterations known during development of mastitis. Similar to SCC, the new DSCC parameter increases with increasing signs of mastitis, which is intuitive and evident.

\section{Practical Application of the DSCC Parameter}

The new Foss DSCC method was developed with the objective of providing more accurate information about the actual udder health status of cows and thus allowing improved mastitis screening in the frame of regular DHI testing programs. The new DSCC parameter is generally seen as a complementary parameter to the well-established SCC.

Data from our initial field trial revealed broad variations (34-79\%) of DSCC percentages in samples with $<400,000$ cells $/ \mathrm{mL}$. Elevated percentages of PMN in samples with low SCC were described previously (Schwarz et al., 2011a,b; Pilla et al., 2012) and interpreted as an early indication of IMI, despite nonsuspiciously low SCC. High proportions of macrophages were previously typically found in milk of healthy mammary glands (Lee et al., 1980; Östensson et al., 1988), indicating that low DSCC values, which express high proportions of macrophages, could signal a good udder health status.

We found variations from 53 to $89 \%$ in samples with SCC >400,000 cells/mL. Whereas high PMN percentages are understood as a clear sign of infection (Paape et al., 1979), elevated proportions of macrophages were described as an indication of chronic mastitis (Leitner et al., 2000). However, apparent differences between the Foss DSCC method and literature methods must be considered, as discussed above.

Several studies (e.g., Dohoo and Meek, 1982; Harmon, 1994) that were done to understand the practical application of SCC by investigating factors affecting the parameter such as infection status, stage of lactation, number of quarters infected, diurnal variations, and day-to-day variation stress the need for further basic research studies in the field of DSCC.

\section{CONCLUSIONS}

The new Foss DSCC method is the first method that enables routine analysis of both DSCC and SCC using untreated individual cow milk samples. The method allows low-cost, reliable, repeatable, and high-throughput determination of the 2 parameters DSCC and SCC simultaneously. Hence, the developed method is suitable to be applied in CMT laboratories for adding more information, thus allowing improved mastitis screening in the frame of DHI testing programs. The new DSCC parameter represents the percentage of PMN and lymphocytes in milk and is thus complementary to the well-established SCC. Further research on the practical application of the DSCC parameter in DHI programs is, however, needed. The complexity of mastitis requires extensive multi-disciplinary longitudinal studies.

\section{ACKNOWLEDGMENTS}

The authors are grateful to Jurgita Sørensen and Mille Bundgaard (Foss Analytical A/S, Hilleroed, Denmark) for their excellent technical assistance. We further thank the various dairy farms that provided us with milk samples as well as the CMT organizations Analis (Le Mans, France), CanWest DHI (Guelph, Canada), Eurofins (Vejen, Denmark), and Milk Test NZ (Hamilton, New Zealand) for their excellent cooperation. The authors further acknowledge Core Facility for Flow Cytometry, Faculty of Health and Medical Sciences, University of Copenhagen, Copenhagen, Denmark, for the collaboration in terms of flow cytometric analyses, in particular cell sorting experiments.

\section{REFERENCES}

Barcina, Y., M. A. Zorraquino, J. Pedauye, G. Ros, and F. Rincón. 1987. Azidiol as a preservative for milk samples. An. Vet 3:65-69.

Barkema, H. W., Y. H. Schukken, T. J. G. M. Lam, M. L. Beiboer, H. Wilmink, G. Benedictus, and A. Brand. 1998. Incidence of clinical mastitis in dairy herds grouped in three categories by bulk milk somatic cell counts. J. Dairy Sci. 81:411-419.

Basu, S., H. M. Campbell, B. N. Dittel, and A. Ray. 2010. Purification of specific cell population by fluorescence activated cell sorting (FACS). JoVE 41:5-8.

Dohoo, I. R., and A. H. Meek. 1982. Somatic cell counts in bovine milk. Can. Vet. J. 23:119-125.

Halasa, T., K. Huijps, O. Osteras, and H. Hogeveen. 2007. Economic effects of bovine mastitis and mastitis management: A review. Vet. Q. 29:18-31.

Harmon, R. J. 1994. Physiology of mastitis and factors affecting somatic cell counts. J. Dairy Sci. 77:2103-2112.

Holm, C. 2012. Method for determining a degree of infection. Patent number: EP2630487. European Patent Register. Accessed Mar. 15, 2017. https://register.epo.org/application?number=EP10768012.

IDF (International Dairy Federation). 2013. Guidelines for the use and interpretation of bovine milk somatic cell count. Bull. IDF $466 / 2013$.

Kehrli, M. E., and D. E. Shuster. 1994. Factors affecting milk somatic cells and their role in health of the bovine mammary gland. J. Dairy Sci. 77:619-627.

Kitchen, B. J. 1981. Review of the progress of dairy science: Bovine mastitis, milk compositional changes and related diagnostic tests. J. Dairy Res. 48:167-188. 
Koess, C., and J. Hamann. 2008. Detection of mastitis in the bovine mammary gland by flow cytometry at early stages. J. Dairy Res. $75: 225-232$.

Lee, C. S., F. B. P. Wooding, and P. Kemp. 1980. Identification properties and differential counts of cell populations using electron microscopy of dry cows secretions, colostrum and milk from normal cows. J. Dairy Res. 47:39-50.

Leitner, G., E. Shoshani, O. Krifucks, M. Chaffer, and A. Saran. 2000 Milk leucocyte population patterns in bovine udder infection of different aetiology. J. Vet. Med. B Infect. Dis. Vet. Public Health 47:581-589.

Li, N., R. Richoux, M. H. Perruchot, M. Boutinaud, J. F. Mayol, and V. Gagnaire. 2015. Flow cytometry approach to quantify the viability of milk somatic cell counts after various physico-chemical treatments. PLoS One 10:e0146071.

Menzies, F. D., D. G. Bryson, T. McCallion, and D. I. Matthews. 1995. Menzies. Vet. Rec. 137:531-536.

Nickerson, S. C. 1989. Immunological aspects of mammary involution. J. Dairy Sci. 72:1665-1678.

Östensson, K., M. Hageltorn, and G. Åström. 1988. Differential cel counting in fraction-collected milk from dairy cows. Acta Vet. Scand. 29:493-500.

Oviedo-Boyso, J., J. J. Valdez-Alarcón, M. Cajero-Juárez, A. OchoaZarzosa, J. E. López-Meza, A. Bravo-Patiño, and V. M. Baizabal-Aguirre. 2007. Innate immune response of bovine mammary gland to pathogenic bacteria responsible for mastitis. J. Infect. 54:399-409.

Paape, M. J., J. Mehrzad, X. Zhao, J. Detilleux, and C. Burvenich. 2002. Defense of the bovine mammary gland by polymorphonuclear neutrophil leukocytes. J. Mammary Gland Biol. Neoplasia 7:109-121.

Paape, M. J., W. P. Wergin, A. J. Guidry, and R. E. Pearson. 1979. Leukocytes - Second line of defense against invading mastitis pathogens. J. Dairy Sci. 62:135-153.

Pilla, R., M. Malvisi, G. Snel, D. Schwarz, S. König, C.-P. Czerny, and R. Piccinini. 2013. Differential cell count as an alternative method to diagnose dairy cow mastitis. J. Dairy Sci. 96:1653-1660.

Pilla, R., D. Schwarz, S. König, and R. Piccinini. 2012. Microscopic differential cell counting to identify inflammatory reactions in dairy cow quarter milk samples. J. Dairy Sci. 95:4410-4420.
Pillai, S. R., E. Kunze, L. M. Sordillo, and B. M. Jayarao. 2001. Application of differential inflammatory cell count as a tool to monitor udder health. J. Dairy Sci. 84:1413-1420.

Pyörälä, S. 2003. Indicators of inflammation in the diagnosis of mastitis. Vet. Res. 34:565-578.

Rivas, A. L., F. W. Quimby, J. Blue, and O. Coksaygan. 2001. Longitudinal evaluation of bovine mammary gland health status by somatic cell counting, flow cytometry, and cytology. J. Vet. Diagn. Invest. 13:399-407.

Sarikaya, H., C. Werner-Misof, M. Atzkern, and R. M. Bruckmaier. 2005. Distribution of leucocyte populations, and milk composition, in milk fractions of healthy quarters in dairy cows. J. Dairy Res. 72:486-492.

Schröder, A. C., and J. Hamann. 2005. The influence of technical factors on differential cell count in milk. J. Dairy Res. 72:153-158.

Schukken, Y. H., D. J. Wilson, F. Welcome, L. Garrison-Tikofsky, and R. N. Gonzalez. 2003. Monitoring udder health and milk quality using somatic cell counts. Vet. Res. 34:579-596.

Schwarz, D., U. S. Diesterbeck, S. König, K. Brügemann, K. Schlez, M. Zschöck, W. Wolter, and C.-P. Czerny. 2011a. Flow cytometric differential cell counts in milk for the evaluation of inflammatory reactions in clinically healthy and subclinically infected bovine mammary glands. J. Dairy Sci. 94:5033-5044.

Schwarz, D., U. S. Diesterbeck, S. König, K. Brügemann, K. Schlez, M. Zschöck, W. Wolter, and C.-P. Czerny. 2011b. Microscopic differential cell counts in milk for the evaluation of inflammatory reactions in clinically healthy and subclinically infected bovine mammary glands. J. Dairy Res. 78:448-455.

Sordillo, L. M., and S. C. Nickerson. 1988. Morphometric changes in the bovine mammary gland during involution and lactogenesis. Am. J. Vet. Res. 49:1112-1120.

Sordillo, L. M., K. Shafer-Weaver, and D. DeRosa. 1997. Immunobiology of the mammary gland. J. Dairy Sci. 80:1851-1865.

van den Borne, B. H. P., T. Halasa, G. van Schaik, H. Hogeveen, and M. Nielen. 2010. Bioeconomic modeling of lactational antimicrobial treatment of new bovine subclinical intramammary infections caused by contagious pathogens. J. Dairy Sci. 93:4034-4044.

Viguier, C., S. Arora, N. Gilmartin, K. Welbeck, and R. O'Kennedy. 2009. Mastitis detection: Current trends and future perspectives. Trends Biotechnol. 27:486-493. 